\title{
SPACE-TIME BLOCK CODED ADAPTIVE MODULATION AIDED OFDM
}

\author{
T. H. Liew and L. Hanzo \\ Dept. of ECS., Univ. of Southampton, SO17 1BJ, UK. \\ Tel: +44-703-593 125, Fax: +44-703-593 045 \\ Email: lh, thl97rdecs.soton.ac.uk http://www-mobile.ecs.soton.ac.uk
}

\begin{abstract}
Space-time block codes provide substantial diversity advantages for multiple transmit antenna systems at a low decoding complexity. In this contribution, we investigate the achievable diversity advantages in the context of adaptive modulation aided turbo coded OFDM. The two-transmitter, one-receiver $\mathrm{G}_{2}$ block space-time coded scheme using no channel coding or gradually increasing rate turbo coding strikes the best trade-off in terms of its overall performance and complexity. Adaptive OFDM performs impressively, when the extra complexity of space-time coding is not affordable, but no adaptive modulation is necessary in conjunction with the more complex multiple transmit and receive antenna associated scenario.
\end{abstract}

\section{INTRODUCTION}

Recently, a range of different transmit diversity techniques have been introduced, in order to provide diversity gain for mobile stations by upgrading the base stations. In [1], Tarokh et al. proposed space-time trellis coding by jointly designing the channel coding, modulation, transmit diversity and the optional receiver diversity schemes. The resultant space-time trellis codes perform extremely well at the cost of high implementational complexity. In addressing the issue of decoding complexity, Alamouti [2] discovered a remarkable space-time block coded scheme for transmissions using two transmit antennas. A simple decoding algorithm was introduced, which can be generalised to an arbitrary number of receive antennas. This space-time block coded scheme is significantly less complex, than space-time trellis coding using two transmit antennas, although there is a loss in performance [3]. Despite the associated slight performance penalty, Alamouti's scheme is appealing in terms of its simplicity and good overall performance. Alamouti's proposal motivated Tarokh et al. $[3,4]$ to generalise the scheme to an arbitrary number of transmit antennas, leading to the concept of generalised space-time block codes.

Adaptive modulation was proposed by Steele and Webb [5], in order to combat the time-variant fading of mobile channels. Recently adaptive modulation was also proposed for OFDM, which was termed Adaptive OFDM (AOFDM) [6,7]. AOFDM exploits the variation of the signal quality both in the time domain as well as in the frequency domain. In this paper, the AOFDM philosophy proposed by Keller et al. [6,7] is extended, in order to exploit the additional advantages of multiple transmit and receive antennas. Furthermore, turbo coding is also employed in order to improve the performance of the system.

\section{SYSTEM OVERVIEW}

In Figure 1, we show the schematic of the turbo-coded and spacetime-coded adaptive OFDM system. Random data bits are generated and encoded by the TC $(2,1,3)$ encoder using an octal generator polynomial of $(7,5)$ and a constraint length of 3 . Various
$\mathrm{TC}(2,1,3)$ coding rates were used in conjunction with the different modulation schemes. The encoded bits were channel interleaved and passed to the modulator. The choice of the modulation scheme to be used by the transmitter for its next AOFDM symbol is determined by the channel quality estimate of the receiver based on the current AOFDM symbol. In this study, we assumed perfect channel quality estimation and perfect signalling of the required AOFDM modem mode of each sub-band based on the channel quality estimate acquired during the current OFDM symbol. Aided by the perfect channel quality estimator, the receiver determines the highest-throughput AOFDM modulation mode to be employed by the transmitter for its next transmission, while maintaining the system's target Bit Error Rate (BER).

Five possible transmission modes were employed in our investigations, which are no transmission (NoTx), Binary Phase Shift Keying (BPSK), Quadrature Phase Shift Keying (QPSK), 16-level Quadrature Amplitude Modulation (16QAM) and 64-level Quadrature Amplitude Modulation (64QAM). In order to simplify the task of signalling the required modulation modes, we employed the sub-band adaptive OFDM transmission scheme proposed by Keller et al. [6,7]. The AOFDM modulated signals were then passed to the encoder of the space-time block code $\mathbf{G}_{2}$ [2]. The space-time encoded signals were AOFDM modulated and transmitted by the corresponding antennas. In this treatise, we investigate the performance of the proposed systems over the indoor Wireless Asynchronous Transfer Mode (WATM) channels of [6]. The WATM system used 512 subcarriers and each AOFDM symbol was extended with a cyclic prefix of length 64 . The sampling rate was $225 \mathrm{Msamples} / \mathrm{s}$ and the carrier frequency was $60 \mathrm{GHz}$. As shown in Figure 2, the shortened WATM channel's impulse re-

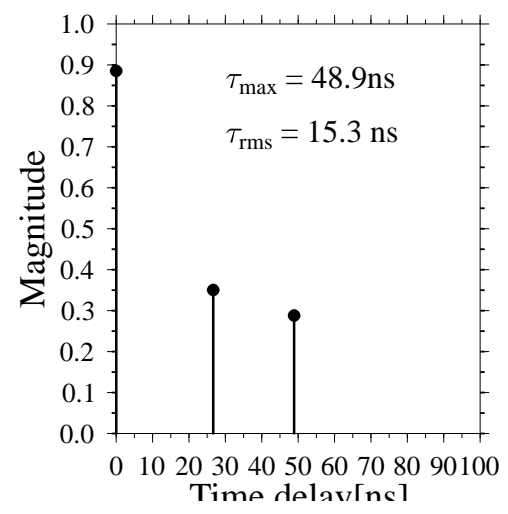

Figure 2: Short WATM channel impulse response [6]. sponse is depicted in Figure 2, where the longest-delay path arrived at a delay of $48.9 \mathrm{~ns}$.

The number of receivers invoked constitutes a design parameters. The received signals were OFDM demodulated and passed to the space-time decoders. Logarithmic Maximum A-Posteriori 


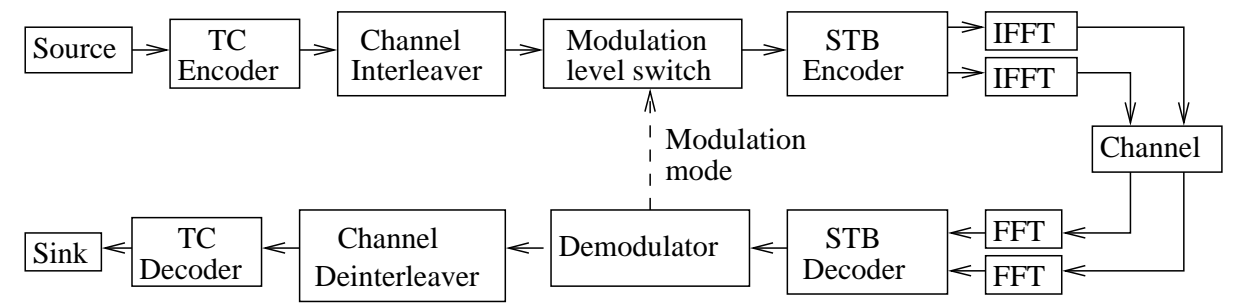

Figure 1: System overview of the turbo-coded and space-time-coded adaptive OFDM.

(Log-MAP) [8] decoding of the received space-time signals was performed, in order to provide soft-outputs for the TC $(2,1,3)$ decoder. Assuming that the demodulator of the receiver has perfect knowledge of the instantaneous channel quality, this information is passed to the transmitter in order to determine its next AOFDM modulation mode allocation. The received bits were then channel deinterleaved and passed to the TC decoder, which again, employs the Log-MAP decoding algorithm $[8,9]$. The decoded bits were finally passed to the sink for calculation of the BER.

\section{SIMULATION RESULTS}

As mentioned earlier, all the AOFDM based simulation results were obtained over the shortened WATM channel shown in Figures 2. Jakes' model [10] was adopted for modelling the fading channels. The average signal power received from each transmitter antenna was the same and all multipath components undergo independent Rayleigh fading. We assume that the receiver has a perfect knowledge of the CIR and perfect signalling of the AOFDM modulation modes is maintained. We note that the above assumptions are unrealistic, yielding the best-case performance, nonetheless, they facilitate the performance comparison of the various techniques under identical circumstances.

\subsection{Space-Time Coded Adaptive OFDM}

In this section, we employ the fixed threshold based AOFDM modem mode selection algorithm, which was also used in [6,7], adapting the technique proposed by Torrance [11] for serial modems. Torrance assumed that the channel quality is constant for all the symbols in a transmission burst, i.e. that the channel's fading envelope varied slowly across the transmission burst. Under these conditions, all the transmitted symbols are modulated using the same modulation mode, chosen according to the predicted SNR. Torrance optimised the modem mode switching thresholds [11] for the target BERs of $10^{-2}$ and $10^{-4}$, which are appropriate for a high-BER speech system and for a low-BER data system, respectively.

\begin{tabular}{|c|c|c|c|c|c|}
\hline System & NoTx & BPSK & QPSK & 16QAM & 64QAM \\
\hline Speech & $-\infty$ & 3.31 & 6.48 & 11.61 & 17.64 \\
\hline Data & $-\infty$ & 7.98 & 10.42 & 16.76 & 26.33 \\
\hline
\end{tabular}

Table 1: Optimised switching levels quoted from [11] for adaptive modulation over Rayleigh fading channels for the speech and data systems, shown in instantaneous channel SNR (dB)

The resulting SNR switching thresholds used for activating a given AOFDM mode in a slowly Rayleigh fading narrowband channel are given in Table 1 for both systems. Assuming perfect channel quality estimation, the instantaneous channel SNR is estimated by the receiver and the information is passed to the AOFDM mode selection switch at the transmitter, as shown in Figure 1 using the system's control channel. This side-information signalling does not constitute a problem, since state-of-the-art wireless systems, such as for example IMT-2000 have a high-rate, low-delay signalling channel. This modem mode signalling feedback information is utilised by the transmitter for selecting the next modulation mode. Specifically, a given AOFDM mode is selected, if the instantaneous channel SNR perceived by the receiver exceeds the corresponding switching levels shown in Figure 1, depending on the target BER.

As mentioned earlier, the proposed adaptation algorithm [11] assumes constant instantaneous channel SNR over the whole transmission burst. However, in the case of an AOFDM system transmitting over frequency selective channels, the channels' quality varies across the different subcarriers. Keller et al. proposed employing the lowest-quality sub-carrier in the sub-band for controlling the adaptation algorithm based on the switching thresholds given in Table 1. Again, this approach significantly simplifies the signalling and therefore it was also adopted in our investigations.

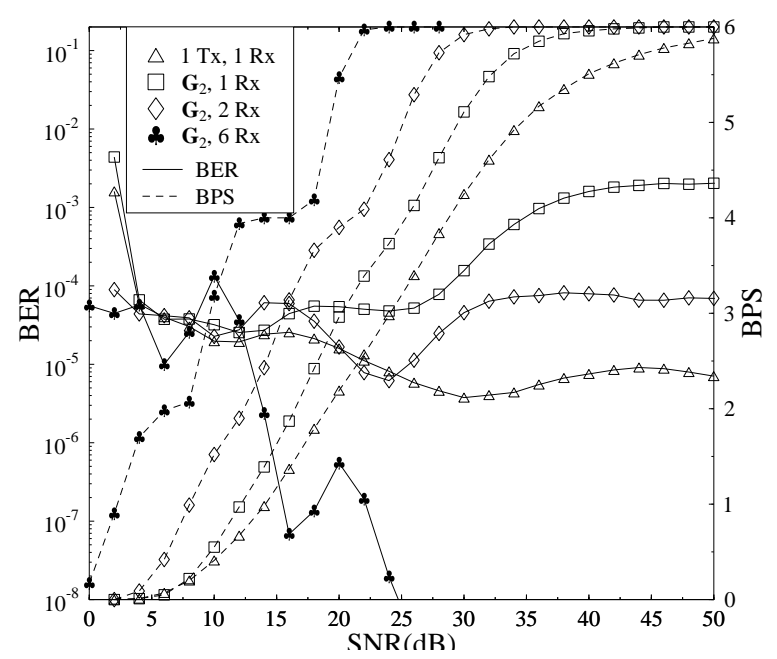

Figure 3: BER and BPS performance of 16 sub-band AOFDM employing the space-time block code $\mathbf{G}_{2}$ using multiple receivers for a target BER of $10^{-4}$ over the shortened WATM channel shown in Figure 2. The switching thresholds are shown in Table 1.

In Figure 3, we show the BER and BPS performance of the 16 sub-band AOFDM scheme employing the space-time block code $\mathbf{G}_{2}$ in conjunction with multiple receivers and a target BER of $10^{-4}$ over the shortened WATM channel shown in Figure 2. Instead of mapping the $\mathbf{G}_{2}$-coded signals $x_{1}$ and $x_{2}$ to two consecutive time instants [2], we mapped them to two consecutive AOFDM subcarriers which experienced near-identical channel transfer factors. The AOFDM switching thresholds are shown in Table 1 . The performance of the conventional AOFDM scheme using no diversity $[6,7]$ is also shown in the figure. From Figure 3, we can see that the BPS performance of the space-time coded AOFDM scheme using one receiver is better, than that of the con- 
ventional AOFDM scheme. The associated performance gain improves, as the throughput increases. At a throughput of 6 BPS, the space-time coded scheme outperforms the conventional scheme by at least $10 \mathrm{~dB}$ in $E_{b} / N_{0}$ terms. However, we notice in Figure 3 that as a secondary effect, the BER performance of the space-time coded AOFDM scheme using one receiver degrades, when we increase the average channel SNR. This problem is due to the interference of the $\mathbf{G}_{2}$-coded transmitted signals $x_{1}$ and $x_{2}$ which were mapped to consecutive AOFDM subcarriers. This phenomenon is caused by the non-identical frequency-domain fading factors of the adjacent subcarriers conveying the signals $x_{1}$ and $x_{2}$ [12]. At high SNRs, predominantly 64QAM was employed. Since the constellation points in 64QAM are densely packed, this modulation mode is more sensitive to the 'cross-talk' of the transmitted signals $x_{1}$ and $x_{2}$. This limited the BER performance to $10^{-3}$ even at high SNRs. However, at SNRs lower than $30 \mathrm{~dB}$ typically more robust modulation modes were employed and hence the target BER of $10^{-4}$ was readily met. We will show in the next section that this problem can be overcome by employing turbo channel coding in the system.

In Figure 3 we also observe that the BER and BPS performance improves, as we increase the number of AOFDM receivers, since the interference between the transmitted signals $x_{1}$ and $x_{2}$ is eliminated. Upon having six AOFDM receivers, the BER of the system drops below $10^{-8}$, when the average channel SNR exceeds $25 \mathrm{~dB}$ and there is no sign of any residual BER. At a throughput of 6 BPS, the space-time coded AOFDM scheme using six receivers outperforms the conventional system by more than $30 \mathrm{~dB}$.

Figure 4 shows the probability of each AOFDM sub-band modulation mode for (a) conventional AOFDM and for space-time coded AOFDM using (b) 1, (c) 2 and (d) 6 receivers over the shortened WATM channel shown in Figure 2. The switching thresholds were optimised for the data system having a target BER of $10^{-4}$ and they are shown in Table 1. By employing multiple transmitters and receivers, we increase the diversity gain and we can see in the figure that this increases the probability of the most appropriate modulation mode at a certain average channel SNR. This is clearly shown by the increased PDF peaks of each modulation mode at different average channel SNRs. As an example, in Figure 4 (d) we can see that there is an almost $100 \%$ probability of transmitting in the QPSK and 16QAM modes at an average channel SNR of approximately $6 \mathrm{~dB}$ and $15 \mathrm{~dB}$, respectively. This strongly suggest that it is a better solution, if fixed modulation based transmission is employed in space-time coded OFDM, provided that we can afford the associated complexity of using six receivers. We shall investigate these issues in more depth at a later stage.

On the other hand, the increased probability of a particular modulation mode at a certain average channel SNR also means that there is less frequent switching amongst the various AOFDM modes. For example, we can see in Figure 4 (b) that the probability of employing 16QAM increased to 0.8 at an average channel SNR of $25 \mathrm{~dB}$ compared to 0.5 in Figure 4 (a). Furthermore, there are almost no BPSK transmissions at $\mathrm{SNR}=25 \mathrm{~dB}$ in Figure 4 (b). This situation might be an advantage in the context of the AOFDM system, since most of the time the system will employ 16QAM and only occasionally switches to the QPSK and 64QAM modulation modes. This can be potentially exploited to reduce the AOFDM modem mode signalling traffic and to simplify the system.

The characteristics of the modem mode probability density functions in Figure 4 in conjunction with multiple transmit antennas can be further explained with the aid of Figure 5. In Figure 5 we show the instantaneous channel SNR experienced by the 512-subcarrier AOFDM symbols for a single-transmitter, singlereceiver scheme and for the space-time block code $\mathbf{G}_{2}$ [2] using one, two and six AOFDM receivers over the shortened WATM
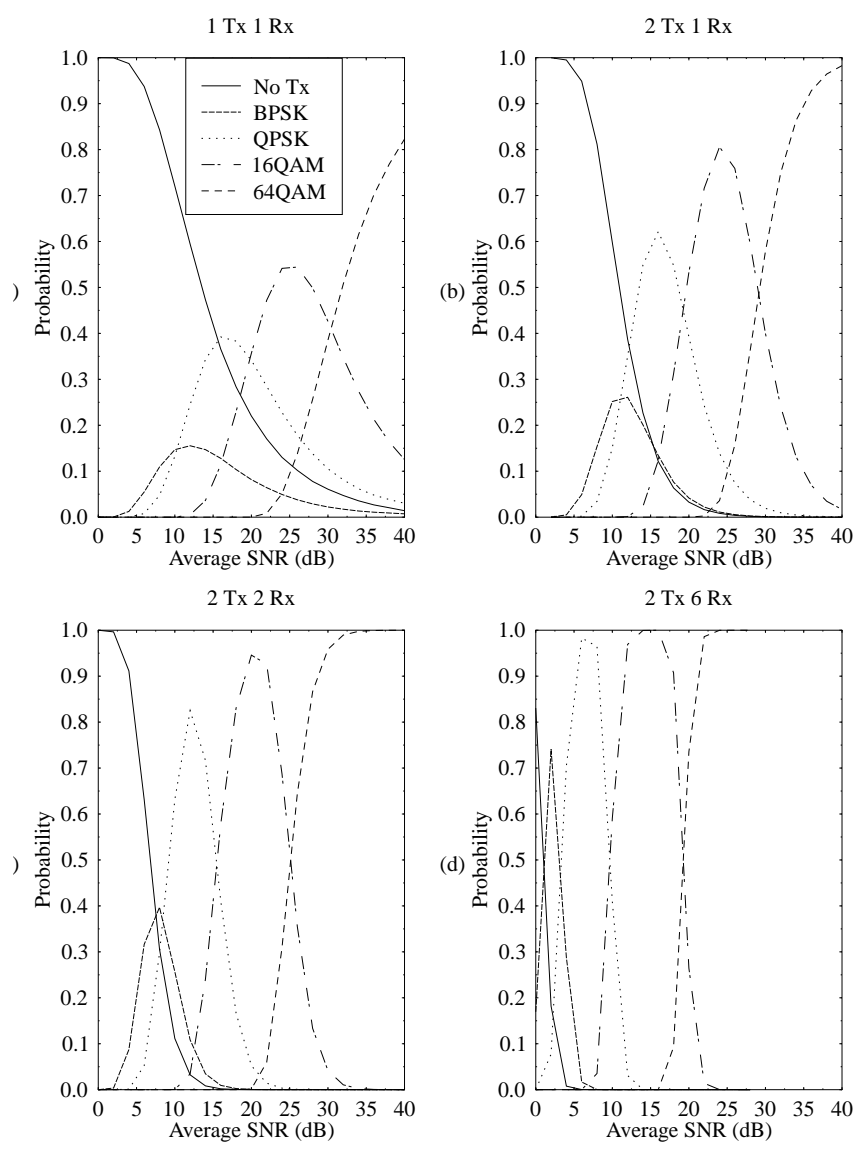

Figure 4: Probability of each modulation mode for (a) conventional AOFDM and for space-time coded AOFDM using (b) 1 , (c) 2 and (d) 6 receivers over the shortened WATM channel shown in Figure 2. The thresholds were optimised are for the data system and they are shown in Table 1. All sub-figures share the legends seen in Figure 4 (a).
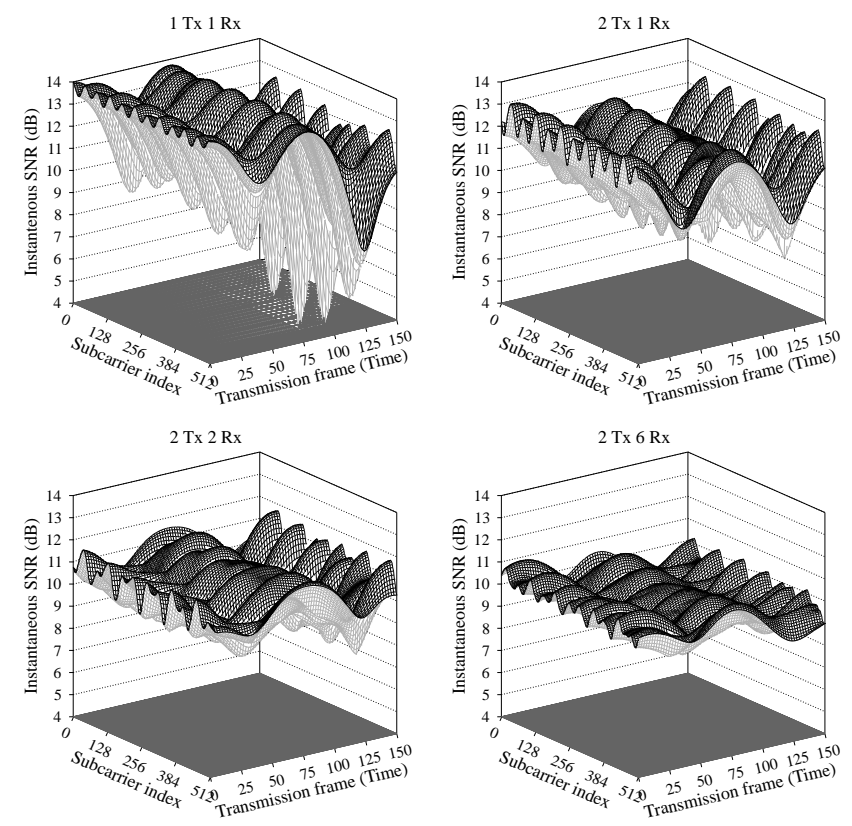

Figure 5: Instantaneous channel SNR of 512-subcarrier OFDM symbols for single-transmitter single-receiver and for the space-time block code $\mathbf{G}_{2}$ using one, two and six receivers over the shortened WATM channel shown in Figure 2. The average channel SNR is $10 \mathrm{~dB}$. 
channel. The average channel SNR is $10 \mathrm{~dB}$. We can see in Figure 5 that the variation of the instantaneous channel SNR for a single transmitter and single receiver is severe. The instantaneous channel SNR may become as low as $4 \mathrm{~dB}$ due to deep fades of the channel. On the other hand, we can see that for the space-time block code $\mathbf{G}_{2}$ using one receiver the variation in the instantaneous channel SNR is significantly less severe. Explicitly, by employing multiple transmit antennas as shown in Figure 5, we have significantly reduced the effect of the channels' deep fades. This is advantageous in the context of adaptive modulation schemes, since higher-order modulation modes can be employed, in order to increase the throughput of the system. However, as we increase the number of AOFDM receivers, i.e. the diversity order, we observe that the variation of the channel becomes more moderate. Effectively, by employing higher-order diversity, the fading channels have been converted to AWGN-like channels, as evidenced by the space-time block code $\mathbf{G}_{2}$ using six receivers shown in Figure 5. Since adaptive modulation only offers advantages over fading channels, we argue that using adaptive modulation might become unnecessary, as the diversity order is increased.
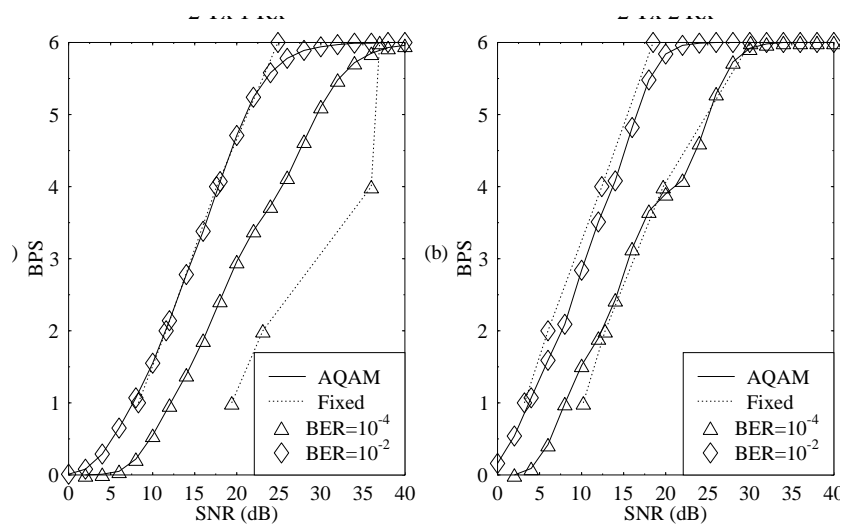

Figure 6: BPS throughput performance comparison between adaptive OFDM and fixed modulation based OFDM using the space-time block code $\mathbf{G}_{2}$ employing (a) one receiver and (b) two receivers over the shortened WATM channel shown in Figure 2.

To elaborate a little further, from Figure 4 and 5 we surmise that fixed modulation schemes might become more attractive, when the diversity order increases, which is achieved in this case by employing more receivers. This is because for a certain average channel SNR, the probability of a particular modulation mode increases. In other words, the fading channel has become an AWGNlike channel, as the diversity order is increased. In Figure 6 we show our throughput performance comparison between AOFDM and fixed modulation based OFDM in conjunction with the spacetime block code $\mathbf{G}_{2}$ employing (a) one receiver and (b) two receivers over the shortened WATM channel. The throughput of fixed OFDM was 1, 2, 4 and 6 BPS and the corresponding $E_{b} / N_{0}$ values were extracted from the associated BER versus $E_{b} / N_{0}$ curves of the individual fixed-mode OFDM schemes. It can be seen from Figure 6 (a) that the throughput performance of the adaptive and fixed OFDM schemes is similar for a $10^{-2}$ target BER system. However, for a $10^{-4}$ target BER system, there is an improvement of 5-10 dB in $E_{b} / N_{0}$ terms at various throughputs for the adaptive OFDM scheme over the fixed OFDM scheme. At high average channel SNRs the throughput performance of both schemes converged, since 64QAM became the dominant modulation mode for AOFDM.

On the other hand, if the number of receivers is increased to two, we can see in Figure 6 (b) that the throughput performance of both adaptive and fixed OFDM is similar for both the $10^{-4}$ and $10^{-2}$ target BER systems. We would expect similar trends, as the number of receivers is increased, since the fading channels become AWGN-like channels. From Figure 6, we conclude that AOFDM is only beneficial for the space-time block code $\mathbf{G}_{2}$ using one receiver in the context of the $10^{-4}$ target BER system.

\subsection{Turbo and Space-Time Coded Adaptive OFDM}

In the previous section we have discussed the performance of spacetime coded adaptive OFDM. Here we extend our study by concatenating turbo coding with the space-time coded AOFDM scheme in order to improve both the BER and BPS performance of the system. The turbo convolutional code $\operatorname{TC}(2,1,3)$ having a constraint length of 3 and octal generator polynomial of $(7,5)$ was employed. Since the system was designed for high-integrity, low-BER data transmission, it was delay non-sensitive. Hence a random turbo interleaver size of approximately 10,000 bits was employed. The random separation channel interleaver of [13] was utilised in order to disperse the bursty channel errors. Again, the Log-MAP [9] decoding algorithm was employed, using eight iterations.

We proposed two different TC coded schemes for the $\mathbf{G}_{2}$ spacetime coded AOFDM system. The first scheme is a fixed half-rate turbo and space-time coded adaptive OFDM system. It achieves a high BER performance, but at the cost of a maximum throughput limited to 3 BPS due to half-rate channel coding. The second one is a variable-rate turbo and space-time coded adaptive OFDM system. This scheme sacrifices the BER performance in exchange for an increased system throughput. Different puncturing patterns are employed for the various code rates $R$. The puncturing patterns were optimised experimentally by simulations. The optimum AOFDM mode switching thresholds were obtained by computer simulations over the shortened WATM channel of Figure 2 and they are shown in Table 2.

\begin{tabular}{|c|c|c|c|c|c|}
\hline & NoTx & BPSK & QPSK & 16QAM & 64QAM \\
\hline \multicolumn{7}{|c|}{ Half-rate TC $(2,1,3)$} \\
\hline Rate & - & 0.50 & 0.50 & 0.50 & 0.50 \\
\hline Thresholds (dB) & $-\infty$ & -4.0 & -1.3 & 5.4 & 9.8 \\
\hline \multicolumn{7}{|c|}{ Variable-rate TC $(2,1,3)$} \\
\hline Rate & - & 0.50 & 0.67 & 0.75 & 0.90 \\
\hline Thresholds (dB) & $-\infty$ & -4.0 & 2.0 & 9.70 & 21.50 \\
\hline
\end{tabular}

Table 2: Coding rates and switching levels $(\mathrm{dB})$ for $\mathrm{TC}(2,1,3)$ and spacetime coded adaptive OFDM over the shortened WATM channel of Figure 2 for a target BER of $10^{-4}$.

In Figure 7, we show the BER and BPS performance of 16 sub-band AOFDM employing the space-time block code $\mathbf{G}_{2}$ concatenated with both half-rate and variable-rate TC $(2,1,3)$ coding at a target BER of $10^{-4}$ over the shortened WATM channel of Figure 2 . We can see in the figure that by concatenating fixed half-rate turbo coding with the space-time coded adaptive OFDM scheme, the BER performance of the system improves tremendously, indicated by a steep dip of the associated BER curve marked by the solid line and diamonds. There is an improvement in the BPS performance as well, exhibiting an $E_{b} / N_{0}$ gain of approximately $5 \mathrm{~dB}$ and $10 \mathrm{~dB}$ at an effective throughput of $1 \mathrm{BPS}$, compared to space-time coded AOFDM and conventional AOFDM, respectively. However, as stated above, the maximum throughput of the system is limited to 3 BPS, since half-rate channel coding was employed. By contrast, in Figure 7 , we can see that at an $E_{b} / N_{0}$ value of about $30 \mathrm{~dB}$ the maximum throughput of the turbo coded and space-time adaptive OFDM system is increased from 3.0 BPS to 5.4 BPS by employing the variable-rate $\mathrm{TC}(2,1,3)$ code. Furthermore, the BPS performance of the variable-rate turbo coded 


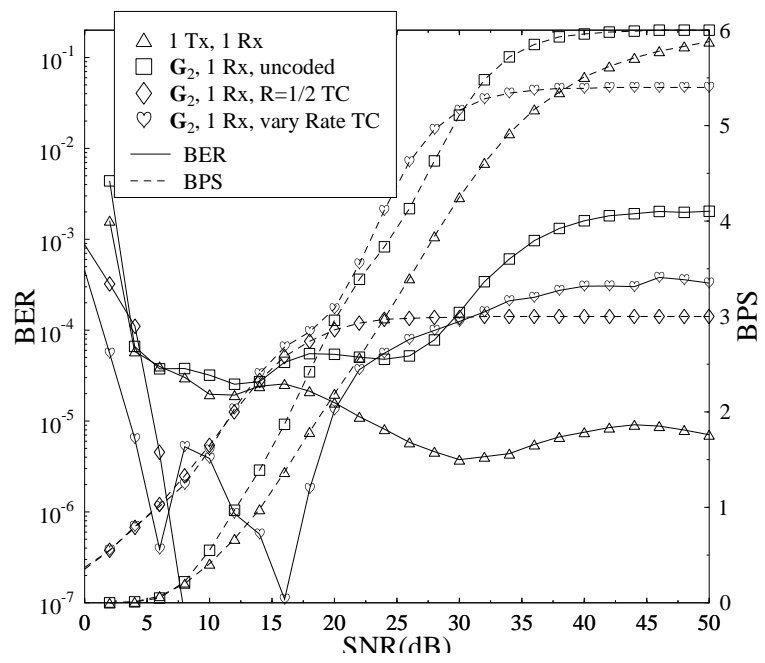

Figure 7: BER and BPS performance of 16 sub-band AOFDM employing the space-time block code $\mathbf{G}_{2}$ concatenated with both half-rate and variable-rate $\mathrm{TC}(2,1,3)$ at a target BER of $10^{-4}$ over the shortened WATM channel shown in Figure 2. The switching thresholds and coding rates are shown in Table 1.

scheme is similar to that of the half-rate turbo coded scheme at average channel SNRs below $15 \mathrm{~dB}$. The BER curve marked by the solid line and clubs drops, as the average channel SNR is increased from $0 \mathrm{~dB}$ to $15 \mathrm{~dB}$. Due to the increased probability of the 64QAM transmission mode, the variable-rate turbo coded scheme was overloaded by the plethora of channel errors introduced by the 64QAM mode. Therefore, we can see in Figure 7 that the BER increases and stabilises at $10^{-4}$. Again, the interference of the signals $x_{1}$ and $x_{2}$ in the context of the space-time block code $\mathbf{G}_{2}$ prohibits further improvements in the BER performance, as the average channel SNR is increased. However, employing the variable-rate turbo codec has reduced the BER floor, as demonstrated by the curve marked by the solid line and squares.

\section{CONCLUSION}

In this paper, we have found that the channel variations have been reduced by employing $\mathbf{G}_{2}$-coding assisted diversity. Therefore, no further benefits accrue from employing AOFDM in conjunction with space-time coding, while AOFDM performs impressively, when the extra complexity of multiple transmitters and receivers is not affordable.

\section{REFERENCES}

[1] V. Tarokh, N. Seshadri and A. R. Calderbank, "Space-Time Codes for High Data Rate Wireless Communication: Performance Criterion and Code Construction", IEEE Transactions on Information Theory, Vol. 44, no. 2 pp. 744-765, March 1998.

[2] S. M. Alamouti, "A Simple Transmit Diversity Technique for Wireless Communications”, IEEE JSAC, pp. 1451-1458, October 1998.

[3] V. Tarokh, H. Jafarkhani and A. R. Calderbank, "Space-Time Block Codes from Orthogonal Designs", IEEE Transactions on Information Theory, pp. 1456-1467, July 1999.

[4] V. Tarokh, H. Jafarkhani and A. R. Calderbank, "Space-Time Block Coding for Wireless Communications: Performance Results", IEEE JSAC, pp. 451-460, March 1999.

[5] W. Webb and R. Steele, "Variable Rate QAM for Mobile Radio", IEEE Transactions on Communications, pp. 2223-2230, Vol. 43, July 1995.
[6] L. Hanzo, W. Webb and T. Keller, "Single- and Multi-carrier Quadrature Amplitude Modulation", John Wiley \& Sons, Ltd, Wessex, England, ISBN 0471492396

[7] T. Keller and L. Hanzo, "Adaptive Orthogonal Frequency Division Multiplexing Schemes", Proceedings of ACTS '98, pp. 794-799, June 1998.

[8] G. Bauch, "Concatenation of Space-Time Block Codes and TurboTCM", Proc IEEE ICC, Vancouver, Canada pp .1202-1206, June 1999.

[9] P. Robertson, E. Villebrun and P. Hoeher, "A Comparison of Optimal and Sub-Optimal MAP Decoding Algorithms Operating in the Log Domain", Proceedings of the International Conference on Communications, pp 1009-1013, June 1995.

[10] W. C. Jakes, "Microwave Mobile Communications", IEEE Press, Piscataway NJ, 1993.

[11] J. Torrance and L. Hanzo, "Performance Upper Bound of Adaptive QAM in Slow Rayleigh Fading Environments", ISPACS 1996, pp 1653-1657, November 1996.

[12] T. Liew, B.-J. Choi and L. Hanzo, "Comparitive Study of Concatenated Turbo Coded and Space-Time block coded as well as SpaceTime Trellis coded OFDM", in Proc. VTC 2001, Rhodes, Greece.

[13] T. Liew, J. Pliquett, B. Yeap, L-L. Yang and L. Hanzo, "Comparative Study of Space Time Block Codes and Various Concatenated Turbo Coding Schemes", PIMRC 2000, London, UK, pp 741-745, 18-21 Sept 2000 . 